\title{
XMM-Newton spectral and timing analysis of the faint millisecond pulsars PSR J0751+1807 and PSR J1012+5307
}

\author{
N. A. Webb ${ }^{1}$, J.-F. Olive ${ }^{1}$, D. Barret ${ }^{1}$, M. Kramer ${ }^{2}$, I. Cognard ${ }^{3}$, and O. Löhmer ${ }^{4}$ \\ ${ }^{1}$ Centre d'Étude Spatiale des Rayonnements, 9 avenue du Colonel Roche, 31028 Toulouse Cedex 04, France \\ 2 University of Manchester, Jodrell Bank Observatory, Macclesfield, Cheshire, SK11 9DL, UK \\ 3 LPCE/CNRS Orléans, 3A avenue de la Recherche Scientifique, 45071 Orléans Cedex 02, France \\ 4 Max-Planck-Institut für Radioastronomie, Auf dem Hügel 69, 53121 Bonn, Germany
}

Received 7 May 2003 / Accepted 2 February 2004

\begin{abstract}
We present XMM-Newton MOS imaging and PN timing data of the faint millisecond pulsars PSR J0751+1807 and PSR J1012+5307. We find 46 sources in the MOS field of view of PSR J0751+1807 searching down to an unabsorbed flux limit of $3 \times 10^{-15} \mathrm{ergs} \mathrm{cm}^{-2} \mathrm{~s}^{-1}(0.2-10.0 \mathrm{keV})$. We present, for the first time, the X-ray spectra of these two faint millisecond pulsars. We find that a power law model best fits the spectrum of PSR J0751+1807, $\Gamma=1.59 \pm 0.20$, with an unabsorbed flux of $4.4 \times 10^{-14} \mathrm{ergs} \mathrm{cm}^{-2} \mathrm{~s}^{-1}(0.2-10.0 \mathrm{keV})$. A power law is also a good description of the spectrum of PSR J1012+5307, $\Gamma=1.78 \pm 0.36$, with an unabsorbed flux of $1.2 \times 10^{-13} \mathrm{ergs} \mathrm{cm}^{-2} \mathrm{~s}^{-1}(0.2-10.0 \mathrm{keV})$. However, a blackbody model cannot be excluded as the best fit to this data. We present some evidence to suggest that both of these millisecond pulsars show pulsations in this X-ray band. We find some evidence for a single broad X-ray pulse for PSR J0751+1807 and we discuss the possibility that there are two pulses per spin period for PSR J1012+5307.
\end{abstract}

Key words. X-rays: stars - stars: pulsars: individual: PSR J0751+1807, PSR J1012+5307 radiation mechanisms: non-thermal - radiation mechanisms: thermal

\section{Introduction}

$\mathrm{X}$-ray emission from millisecond pulsars (MSPs) is thought to be from charged relativistic particles accelerated in the pulsar magnetosphere (non-thermal emission indicated by a hard power-law spectrum and sharp pulsations) and/or thermal emission from hot polar caps and/or emission from a pulsar driven synchrotron nebula or interaction of relativistic pulsar winds with either a wind from a close companion star or the companion star itself (see e.g. Becker \& Aschenbach 2002, for a more thorough review). Until now, X-ray pulsations and spectra have often not been observable for faint MSPs e.g. PSR J0751+1807 (Becker et al. 1996) and PSR J1012+5307 (Halpern \& Wang 1997). Thus it has been difficult to discriminate between competing neutron star models. However, taking advantage of the large collecting area of XMM-Newton (Jansen et al. 2001), it is becoming possible to observe not only the X-ray spectra but also put a limit on the presence of X-ray pulsations of these faint MSPs.

We have observed two faint MSPs with XMM-Newton. PSR J0751+1807 was first detected in an EGRET source error box, in September 1993 (Lundgren et al. 1993), using the radio telescope at Arecibo. Lundgren et al. (1995) combined

Send offprint requests to: N. A. Webb, e-mail: Natalie.Webb@cesr.fr the mass function, eccentricity, orbital size and age of the pulsar, determined from radio data, to predict the expected type of companion star to the millisecond pulsar. They proposed that the secondary star is a helium white dwarf, with a mass between $0.12-0.6 M_{\odot}$, in a $6.3 \mathrm{~h}$ orbit with the pulsar. They determined a $3.49 \mathrm{~ms}$ pulse period, but from the period derivatives, the spin down energy indicated that the pulsar is not the source of the $\gamma$-rays that were originally detected by EGRET. PSR J0751+1807 was subsequently detected in the soft X-ray domain by Becker et al. (1996), using the ROSAT PSPC. However, there were too few counts detected to fit a spectrum or detect pulsations. Using the HI survey of Stark et al. (1992), they deduced an interstellar absorption of $4 \times 10^{20} \mathrm{~cm}^{-2}$. Then using their estimated counts and assuming a powerlaw spectrum of $\mathrm{d} N / \mathrm{d} E \propto E^{-2.5}$, they determined an unabsorbed flux of $1 \times 10^{-13} \mathrm{erg} \mathrm{cm}^{-2} \mathrm{~s}^{-1}(0.1-2.4 \mathrm{keV})$. The corresponding X-ray luminosity was then calculated to be $L_{\mathrm{x}}=4.7 \times 10^{31} \mathrm{erg} \mathrm{s}^{-1}$, for a distance of $2 \mathrm{kpc}$. The distance they used was calculated using the radio dispersion measure and the model of Taylor \& Cordes (1993) for the galactic distribution of free electrons.

PSR J1012+5307, a 5.26 ms pulsar, was discovered by Nicastro et al. (1995) during a survey for short period pulsars conducted with the 76-m Lovell radio telescope. From the dispersion measure they found a distance of $0.52 \mathrm{kpc}$. Callanan et al. (1998), and references therein, confirmed a 
white dwarf secondary of mass $0.16 \pm 0.02 M_{\odot}$ in a $14.5 \mathrm{~h}$ circular orbit with the pulsar. Halpern (1996) associated a faint $\left(L_{\mathrm{x}} \approx 2.5 \times 10^{30} \mathrm{ergs} \mathrm{s}{ }^{-1}, 0.1-2.4 \mathrm{keV}\right)$ X-ray source detected with the ROSAT PSPC $(80 \pm 24$ photons), with the radio MSP PSR J1012+5307. However, the number of photons was insufficient to determine a spectrum or any pulsations.

In this work we present the X-ray spectrum of both PSR J0751+1807 and PSR J1012+5307 for the first time. We also present some evidence for X-ray pulsation from both of these faint pulsars and we compare their nature with other millisecond pulsars, e.g. PSR J0437-4715 (Becker \& Trümper 1993; Zavlin \& Pavlov 1998; Zavlin et al. 2002).

\section{Observations and data reduction}

PSR J0751+1807 was observed by XMM-Newton on October 1, 2000. The observations spanned 38 ks (MOS cameras) and $36.8 \mathrm{ks}$ (PN camera), but a soft proton flare affected approximately $8 \mathrm{ks}$ of these observations. The second of our faint MSPs, PSR J1012+5307, was observed on April 19, 2001. The MOS observations lasted $20.8 \mathrm{ks}$ and the PN observations $19.2 \mathrm{ks}$. However the whole observation was strongly affected by a soft proton flare. The MOS data were reduced using version 5.4.1 of the XMM-Newton SAS (Science Analysis Software). For the PN data we took advantage of the development track version of the SAS. Improvements have been made to the oal (ODF (Observation Data File) access layer) task (version 3.106) to correct for spurious and wrong values, premature increments, random jumps and blocks of frames stemming from different quadrants in the timing data in the PN auxiliary file, as well as correcting properly for the onboard delays (Kirsch et al. 2003). Indeed several spurious jumps were corrected using this version. We have verified that this version improved our timing solution using the pulsar PSR J0218+4232 (see Webb et al. 2004).

We employed the MOS cameras in the full frame mode, using a thin filter (see Turner et al. 2001). The MOS data were reduced using "emchain" with "embadpixfind" to detect the bad pixels. The event lists were filtered, so that $0-12$ of the predefined patterns (single, double, triple, and quadruple pixel events) were retained and the high background periods were identified by defining a count rate threshold above the low background rate and the periods of higher background counts were then flagged in the event list. We also filtered in energy. We used the energy range $0.2-10.0 \mathrm{keV}$, as recommended in the document "EPIC Status of Calibration and Data Analysis" (Kirsch et al. 2002). The event lists from the two MOS cameras were merged, to increase the signal-to-noise.

The PN camera was also used with a thin filter, but in timing mode which has a timing resolution of $30 \mu$ s (Strüder et al. 2001). The PN data were reduced using the "epchain" of the SAS. Again the event lists were filtered, so that $0-4$ of the predefined patterns (single and double events) were retained, as these have the best energy calibration. We also filtered in energy. The document "EPIC Status of Calibration and Data Analysis" (Kirsch et al. 2002) recommends use of PN timing data above $0.5 \mathrm{keV}$, to avoid increased noise. We used the data between $0.6-10.0 \mathrm{keV}$ as this had the best signal-to-noise.
The times of the events were then converted from times expressed in the local satellite frame to Barycentric Dynamical Time, using the task "barycen" and the coordinates derived from observing the pulsar 4-8 times per month, over years, with the radio telescope at Nançay, France, by one of us (IC), for PSR J0751+1807. For PSR J1012+5307 we used the results from the high-precision timing observations taken since October 1996, using the 100-m Effelsberg radio telescope and the 76-m Lovell telescope by two of us (MK and OL).

\section{PSR J0751+1807}

\subsection{Spectral analysis}

We extracted the MOS spectra of PSR J0751+1807 using an extraction radius of $\sim 1^{\prime}$ and rebinned the data into $15 \mathrm{eV}$ bins. We used a similar neighbouring surface, free of X-ray sources to extract a background file. We used the SAS tasks "rmfgen" and "arfgen" to generate a "redistribution matrix file" and an "ancillary response file". We binned the data to contain at least 20 counts/bin. The PN data were extracted in a similar way, following the usual XMM-Newton timing data procedure. The data in the RAWY direction were binned into a single bin and the spectrum was extracted using a rectangle of $1 \times 3$ pixels, which included all the photons from the pulsar, see e.g. Kuster et al. (2002). The background spectrum was extracted from a similar neighbouring surface, free of X-ray sources. Again we used the SAS tasks "rmfgen" and "arfgen" to generate a "redistribution matrix file" and an "ancillary response file". We then used Xspec (version 11.1.0) to fit the spectrum. We tried to fit simple models to the combined PN and MOS spectra. We found the model fits as given in Table 1 for the spectrum between $0.2-10.0 \mathrm{keV}$, when the $N_{\mathrm{H}}$ was frozen at $4 \times 10^{20} \mathrm{~cm}^{-2}$, (see Becker et al. 1996). The best fitting spectrum is a single power law, with a similar photon index to the X-ray spectrum of other millisecond pulsars e.g. PSR B1821-24 (Saito et al. 1997). The spectrum of PSR J0751+1807, plotted with the power law fit, can be seen in Fig. 1. Allowing the $N_{\mathrm{H}}$ to vary gives values compatible with the above values. We determine an unabsorbed flux of $4.4 \times 10^{-14} \mathrm{ergs} \mathrm{cm}^{-2} \mathrm{~s}^{-1}(0.2-10.0 \mathrm{keV})$.

\subsection{Timing analysis}

We have reduced and analysed the timing data in the same way as the timing analysis that we carried out on the MSP PSR J0218+4232 (Webb et al. 2004), which is an MSP that is known to show pulsations in both the radio and X-rays. We corrected the timing data for the orbital movement of the pulsar and the data were folded on the radio ephemeris, see Table 2, taking into account the time-delays due to the orbital motion. We used the data between $0.6-7.0 \mathrm{keV}$, as we found that the majority of the emission from PSR J0751+1807 was in this energy band (see Sect. 3.1) and thus the signal-to-noise in this band was the best. We tested the hypothesis that there is no pulsation in the MSP PSR J0751+1807. We searched frequencies at and around the expected frequency and we found the largest peak in the $\chi_{v}^{2}$ versus change in frequency from the expected frequency at the expected value, see Fig. 2 where we 
Table 1. The best model fits to the MOS spectra of the source 52, and the MOS and PN spectra of PSR J0751+1807 and PSR J1012+5307. For PSR J0751+1807 the interstellar absorption $\left(N_{\mathrm{H}}\right)$ was fixed at $4 \times 10^{20} \mathrm{~cm}^{-2}$. For PSR J1012+5307 the $N_{\mathrm{H}}$ was fixed at $7 \times 10^{19} \mathrm{~cm}^{-2}$. For source $52, z$ was fixed at 0.255 . All the errors shown are $1 \sigma$ confidence limits. The fluxes and luminosities are given for the $0.2-10.0 \mathrm{keV}$ band.

\begin{tabular}{|c|c|c|c|c|c|c|c|c|c|}
\hline Object & $\begin{array}{l}\text { Spectral } \\
\text { model }\end{array}$ & $\begin{array}{c}N_{\mathrm{H}}\left(\mathrm{cm}^{-2}\right) \\
\left(\times 10^{22}\right)\end{array}$ & $\begin{array}{c}k T \\
(\mathrm{keV})\end{array}$ & $\begin{array}{c}\text { Photon } \\
\text { Index }\end{array}$ & Abundance & $\chi_{v}^{2}$ & d.o.f. & $\begin{array}{l}\text { Flux }\left(\times 10^{-13}\right) \\
\left(\mathrm{ergs} \mathrm{cm}^{-2} \mathrm{~s}^{-1}\right)\end{array}$ & $\begin{array}{c}\text { Luminosity } \\
\left(\mathrm{ergs} \mathrm{s}^{-1}\right)\end{array}$ \\
\hline \multirow[t]{3}{*}{ PSR J0751+1807 } & Power law & 0.04 & - & $1.59 \pm 0.20$ & - & 1.33 & 14 & 0.44 & $2.1 \times 10^{31}$ \\
\hline & Bremsstrahlung & 0.04 & $12.36 \pm 12.31$ & - & & 1.52 & 14 & & \\
\hline & Blackbody & 0.04 & $0.32 \pm 0.04$ & - & & 2.21 & 14 & & \\
\hline \multirow[t]{3}{*}{ Source 52} & Power law & $0.13 \pm 0.06$ & - & $1.46 \pm 0.13$ & - & 0.86 & 32 & 2.0 & $1.9 \times 10^{43}$ \\
\hline & Bremsstrahlung & $0.09 \pm 0.05$ & $17.31 \pm 10.42$ & - & & 0.87 & 32 & & \\
\hline & Raymond Smith & $0.07 \pm 0.04$ & $17.63 \pm 12.78$ & - & $0.16 \pm 1.20$ & 0.89 & 31 & & \\
\hline \multirow[t]{3}{*}{ PSR J1012+5307 } & Power law & 0.007 & - & $1.78 \pm 0.36$ & - & 1.27 & 9 & 1.2 & $3.9 \times 10^{30}$ \\
\hline & Bremsstrahlung & 0.007 & $1.95 \pm 1.45$ & - & & 1.29 & 9 & & \\
\hline & Blackbody & 0.007 & $0.26 \pm 0.04$ & - & & 1.37 & 9 & & \\
\hline
\end{tabular}

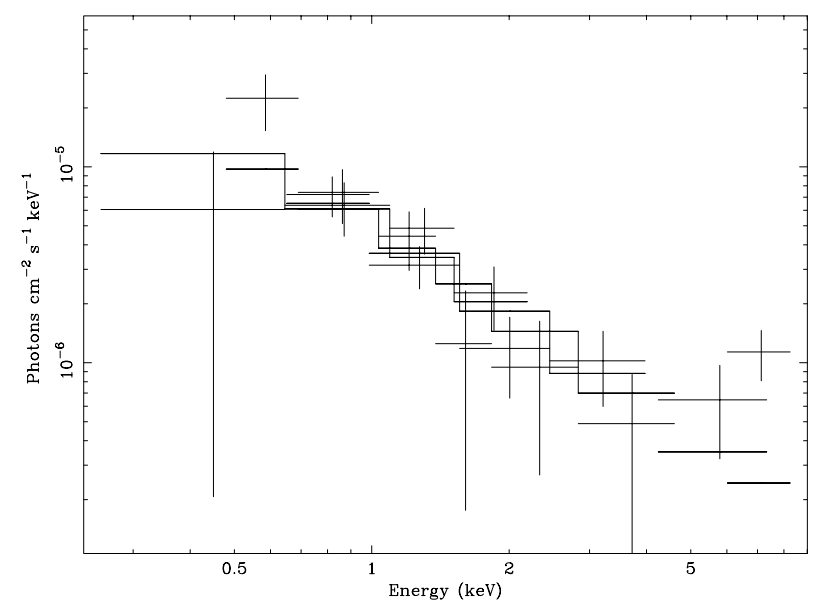

Fig. 1. The combined MOS and PN spectrum of PSR J0751+1807 fitted with a power law model. The fit parameters can be found in Table 1.

have taken the resolution of the data (n) and plotted $4 \mathrm{n}$ bins in the range $-1.75 \times 10^{-4}<\Delta f<1.75 \times 10^{-4}$. Testing the significance of the peak (Buccheri et al. 1985), we find that it is significant at $1.7 \sigma$. However, as this is the largest peak when searching 700 frequencies about the expected frequency and it falls at the expected value, we tried to fold the data on this frequency. The folded lightcurve $(0.6-7.0 \mathrm{keV})$, counts versus phase, is shown in Fig. 3. We find one broad pulse per period. Fitting the lightcurve with a Lorentzian (as Kuiper et al. 2002a) we find that the FWHM of the pulse is $\delta \phi_{1}=0.31 \pm 0.11$, centred at phase $\phi_{1}=0.38 \pm 0.04$ (errors are $90 \%$ confidence). Fitting with a Gaussian gives similar results. Using a $Z_{2}^{2}$ test (Buccheri et al. 1983), which is independent of binning, we determine a value of 5.5 , which corresponds to a probability that the pulse-phase distribution deviates from a statistically flat distribution of 0.94 . The pulsed percentage is $52 \pm 8 \%$.

Taking the $261 \pm 60$ counts above the background in our observation, we are also able to analyse the variations in the lightcurves from different energy bands. We chose the energy bands $0.6-1.0 \mathrm{keV}, 1.0-2.0 \mathrm{keV}$ and $2.0-7.0 \mathrm{keV}$. The data

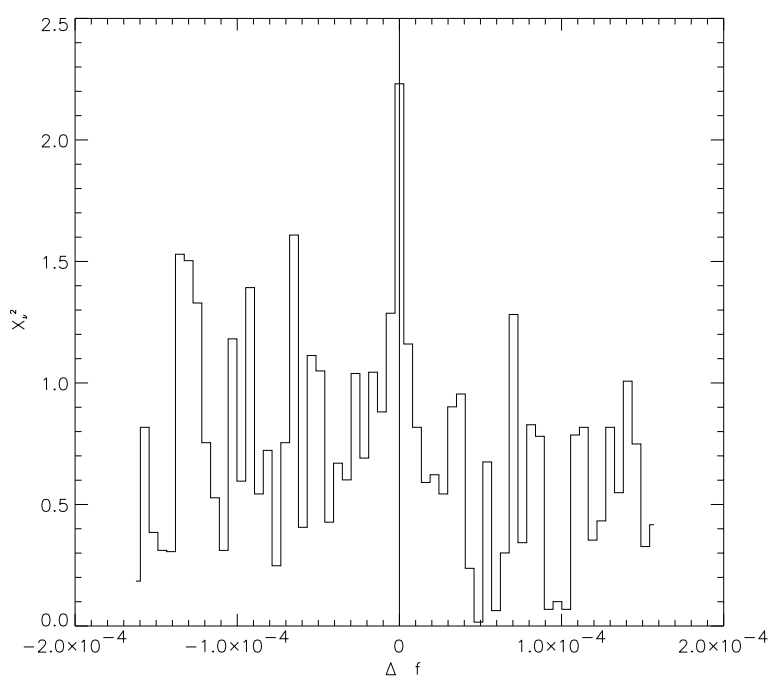

Fig. 2. $\chi_{v}^{2}$ versus change in frequency from the expected pulsation frequency (shown as the solid vertical line at $\Delta f=0.0$ ) for PSR J0751+1807.

Table 2. Ephemeris of PSR J0751+1807 from the Nançay radio timing data.

\begin{tabular}{ll}
\hline \hline Parameter & Value \\
\hline Right ascension $(\mathrm{J} 2000)$ & $07^{\mathrm{h}} 51^{\mathrm{m}} 09^{\mathrm{S}} .156312$ \\
Declination $(\mathrm{J} 2000)$ & $18^{\circ} 07^{\prime} 38^{\prime \prime} 590620$ \\
Period $(P)$ & $0.003478770781560571 \mathrm{~s}$ \\
Period derivative $(\dot{P})$ & $0.726912 \times 10^{-20} \mathrm{~s} \mathrm{~s}^{-1}$ \\
Second period derivative $(\ddot{P})$ & $2.48928 \times 10^{-30} \mathrm{~s} \mathrm{~s}^{-2}$ \\
Frequency $(v)$ & $287.457858811 \mathrm{~Hz}$ \\
Frequency derivative $(\dot{v})$ & $-6.0066207 \times 10^{-16} \mathrm{~Hz} \mathrm{~s}^{-1}$ \\
Second frequency derivative $(\ddot{v})$ & $-2.0569423 \times 10^{-25} \mathrm{~Hz} \mathrm{~s}^{-2}$ \\
Epoch of the period (MJD) & 49301.5 \\
Orbital period & $22735.664643 \mathrm{~s}$ \\
$a$. sin $i$ & 0.39660728 \\
Eccentricity & 0.0000566981 \\
Time of ascending node (MJD) & 49460.430540 \\
\hline
\end{tabular}




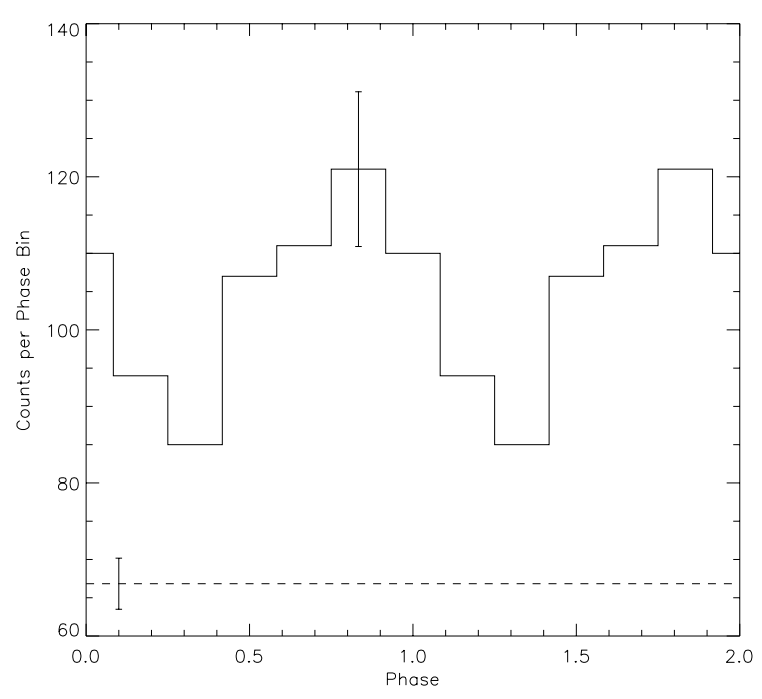

Fig. 3. Lightcurve of PSR J0751+1807 folded on the radio ephemeris and binned into 6 bins, each of $0.58 \mathrm{~ms}$. Two cycles are shown for clarity. A typical $\pm 1 \sigma$ error bar is shown. The dashed line shows the background level, where the error bar represents the $\pm 1 \sigma$ error.

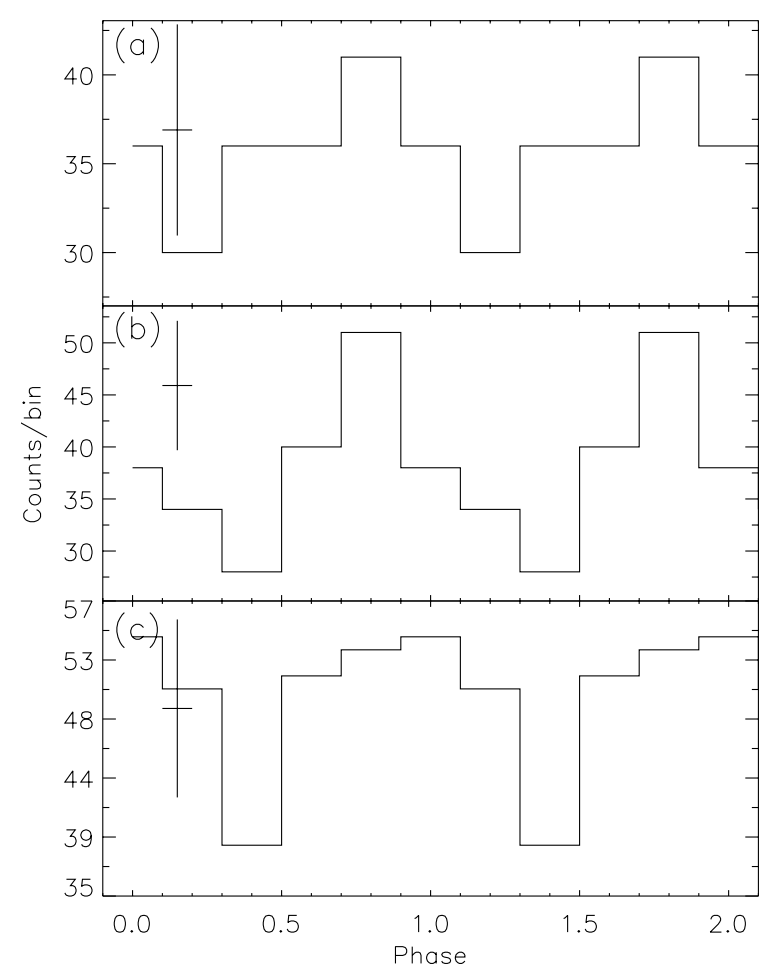

Fig. 4. Lightcurves of PSR J0751+1807 from different energy bands, folded on the radio ephemeris and binned into 5 bins. Two cycles are shown for clarity. a) $0.6-1.0 \mathrm{keV}$; b) $1.0-2.0 \mathrm{keV}$; c) $2.0-7.0 \mathrm{keV}$. A typical $\pm 1 \sigma$ error bar is shown.

were folded as before and binned into 5 bins, where two cycles in phase are presented for clarity. These lightcurves can be seen in Fig. 4. As can also be seen from the spectral fitting, see Sect. 3.1, PSR J0751+1807 emits mostly at lower energies. The lightcurves do not vary dramatically, although the peak may be slightly narrower at lower energies. The pulsed percentage is possibly less at lower energies. We find $32 \pm 16 \%$ for each of

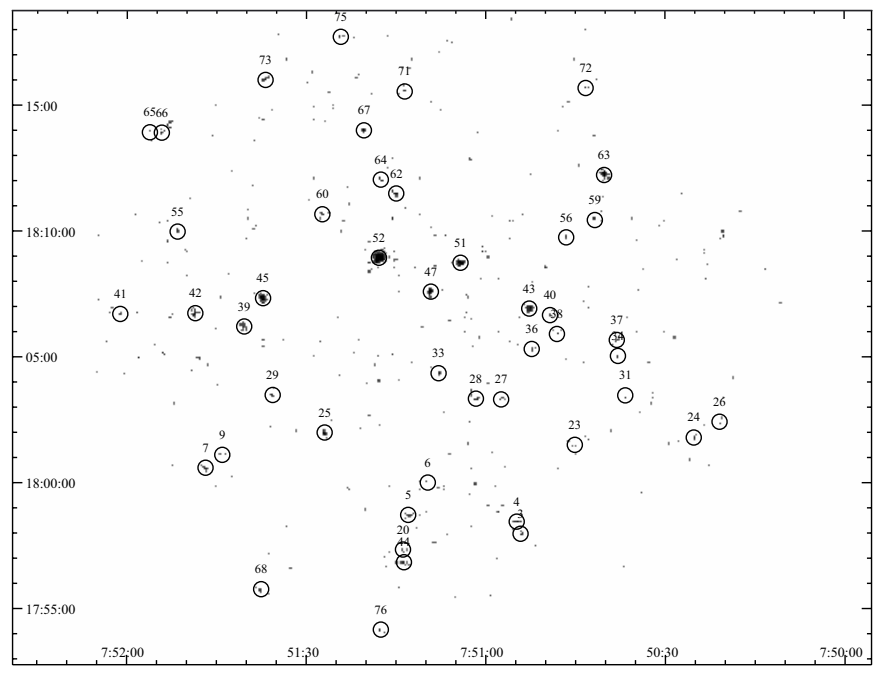

Fig. 5. The MOS field of view. The sources detected with a likelihood $>4.5 \sigma$ are encircled and labelled with a number. These numbers correspond to the source identification numbers in Table 3 . PSR J0751+1807 is labelled 47.

the energy bands: $0.6-1.0 \mathrm{keV}$; and $1.0-2.0 \mathrm{keV}$ and $55 \pm 23 \%$ for the $2.0-7.0 \mathrm{keV}$ band.

\subsection{The MOS field of view}

We have detected 46 sources in total using the task "emldetect", with a maximum detection likelihood threshold of $4.5 \sigma$ and ignoring those sources not found on both cameras (unless they lay outside the FOV or were in a chip gap). These sources can be seen in Fig. 5 and details such as their position, count rates and likelihood of detection can be found in Table 3. PSR J0751+1807 is labelled as source 47. ROSAT detected 10 sources in the XMM-Newton FOV (Becker et al. 1996). We calculate an unabsorbed flux limit of our observation of $3.0 \times 10^{-15} \mathrm{ergs} \mathrm{cm}^{-2} \mathrm{~s}^{-1}(0.2-10.0 \mathrm{keV})$, using PIMMS (Mission Count Rate Simulator) version 3.3a, with a power law, $\Gamma=2$ (as in Hasinger et al. 2001).

The brightest source in the field of view is source 52, which was not detected by ROSAT, where the deepest observation had a detection limit of $1.5 \times 10^{-14} \mathrm{ergs} \mathrm{cm}^{-2} \mathrm{~s}^{-1}$ $(0.2-10.0 \mathrm{keV})$. We detect this source with an unabsorbed flux of $2.0 \times 10^{-13} \mathrm{ergs} \mathrm{cm}^{-2} \mathrm{~s}^{-1}(0.2-10.0 \mathrm{keV})$, more than 13 times brighter than the ROSAT detection limit. This source has been observed as a part of the AXIS (An XMM-Newton International Survey) project (see http://www.ifca.unican.es/ xray/AXIS/ and also Barcons et al. 2002). The source identified as the optical counterpart of our source 52 in the $i$-band photometry is elongated and the spectrum shows calcium $\mathrm{H}$ and $\mathrm{K}$ lines and the $G$-band. From the spectral line shifts, a redshift of $z=0.255$ has been determined by the AXIS group and they propose that this source could be a galaxy. We extracted the MOS spectra of source 52 in the same way as described in Sect. 3.1. We have fitted the spectrum with the extra-galactic origin in mind and the results can be found in Table 1 . The data fitted with a power law model can be found in Fig. 6. We investigated both 
Table 3. X-ray sources in the PSR J0751+1807 MOS field of view. Information includes the source identification number, right ascension and declination of the source, count rate and detection likelihood. The detection likelihood value is the value given by "emldetect" for detection in the two MOS cameras, corrected for the coding error in versions 4.11 .15 and earlier.

\begin{tabular}{|c|c|c|c|c|}
\hline Src & RA (2000) & $\overline{D e c}(2000)$ & Counts/s & Like- \\
\hline ID & $\mathrm{h} \quad \mathrm{m} \quad \mathrm{s}$ & $\circ, "$, & $\times 10^{-3}$ & lihood \\
\hline 3 & 75054.1 & 175759.3 & $5.6 \pm 0.8$ & 62 \\
\hline 4 & 75054.8 & 175827.9 & $5.6 \pm 0.8$ & 63 \\
\hline 5 & 75112.9 & 175844.3 & $4.4 \pm 0.6$ & 53 \\
\hline 13 & 7519.7 & 1801.04 & $3.3 \pm 0.5$ & 40 \\
\hline 17 & 75146.8 & 18036.0 & $6.4 \pm 0.8$ & 99 \\
\hline 19 & 75144.0 & 1817.05 & $3.8 \pm 0.7$ & 32 \\
\hline 20 & 75113.8 & 175721.3 & $3.7 \pm 0.7$ & 23 \\
\hline 23 & 75045.1 & 18130.8 & $3.5 \pm 0.6$ & 31 \\
\hline 24 & 75025.2 & 18148.4 & $6.6 \pm 1.1$ & 40 \\
\hline 25 & 75126.9 & 1820.08 & $6.4 \pm 0.6$ & 134 \\
\hline 26 & 75020.9 & 18225.6 & $5.3 \pm 1.0$ & 34 \\
\hline 27 & 75057.4 & 18319.2 & $2.8 \pm 0.4$ & 39 \\
\hline 28 & 7511.6 & 18320.7 & $3.7 \pm 0.5$ & 65 \\
\hline 29 & 75135.6 & 18329.6 & $4.9 \pm 0.6$ & 64 \\
\hline 31 & 75036.6 & 18328.8 & $3.0 \pm 0.7$ & 18 \\
\hline 33 & 7517.9 & 18421.4 & $3.6 \pm 0.4$ & 67 \\
\hline 34 & 75037.9 & 1852.7 & $3.3 \pm 0.6$ & 28 \\
\hline 36 & 75052.3 & 18519.1 & $2.6 \pm 0.4$ & 32 \\
\hline 37 & 75038.0 & 18540.8 & $4.0 \pm 0.6$ & 48 \\
\hline 38 & 75048.1 & 18555.1 & $3.3 \pm 0.5$ & 60 \\
\hline 39 & 75140.4 & 18612.9 & $9.6 \pm 1.1$ & 106 \\
\hline 40 & 75049.2 & 18639.9 & $2.8 \pm 0.4$ & 43 \\
\hline 41 & 7521.1 & 18642.6 & $5.3 \pm 0.9$ & 38 \\
\hline 42 & 75148.5 & 18644.6 & $8.3 \pm 0.8$ & 141 \\
\hline 43 & 75052.7 & 18655.7 & $6.9 \pm 0.6$ & 202 \\
\hline 44 & 75113.6 & 175650.7 & $6.1 \pm 0.9$ & 56 \\
\hline 45 & 75137.2 & 18720.4 & $9.6 \pm 0.7$ & 255 \\
\hline 47 & 7519.1 & 18736.3 & $8.9 \pm 0.6$ & 327 \\
\hline 51 & 7514.2 & 18845.0 & $12.6 \pm 0.7$ & 466 \\
\hline 52 & 75117.8 & 18856.4 & $36.3 \pm 1.2$ & 2017 \\
\hline 55 & 75151.5 & 18959.2 & $4.2 \pm 0.6$ & 44 \\
\hline 56 & 75046.5 & 18945.2 & $4.6 \pm 0.7$ & 56 \\
\hline 59 & 75041.7 & 181026.7 & $3.0 \pm 0.5$ & 37 \\
\hline 60 & 75127.3 & 181040.2 & $3.0 \pm 0.5$ & 41 \\
\hline 62 & 75114.9 & 181130.0 & $3.1 \pm 0.4$ & 56 \\
\hline 63 & 75040.1 & 181214.3 & $17.2 \pm 1.2$ & 355 \\
\hline 64 & 75117.5 & 18122.9 & $2.7 \pm 0.4$ & 47 \\
\hline 65 & 75156.1 & 181355.7 & $5.4 \pm 1.0$ & 37 \\
\hline 66 & 75154.1 & 181354.9 & $8.6 \pm 1.2$ & 73 \\
\hline 67 & 75120.3 & 18140.7 & $6.6 \pm 0.6$ & 117 \\
\hline 68 & 75137.5 & 175546.9 & $6.9 \pm 1.1$ & 53 \\
\hline 71 & 75113.5 & 181533.4 & $3.7 \pm 0.6$ & 40 \\
\hline 72 & 75043.2 & 181541.3 & $4.4 \pm 0.8$ & 28 \\
\hline 73 & 75136.8 & 18160.5 & $6.3 \pm 0.8$ & 96 \\
\hline 75 & 75124.2 & 181743.5 & $5.2 \pm 0.8$ & 38 \\
\hline 76 & 75117.5 & 175410.7 & $6.1 \pm 1.0$ & 41 \\
\hline
\end{tabular}

models with the redshift frozen at 0.255 and also models with the redshift as a free parameter. We found that the fits for the frozen and the variable redshift $(z)$ were the same within the error bars, hence we present only one set of results, those where the redshift was fixed at 0.255 . We find a reasonably hard X-ray

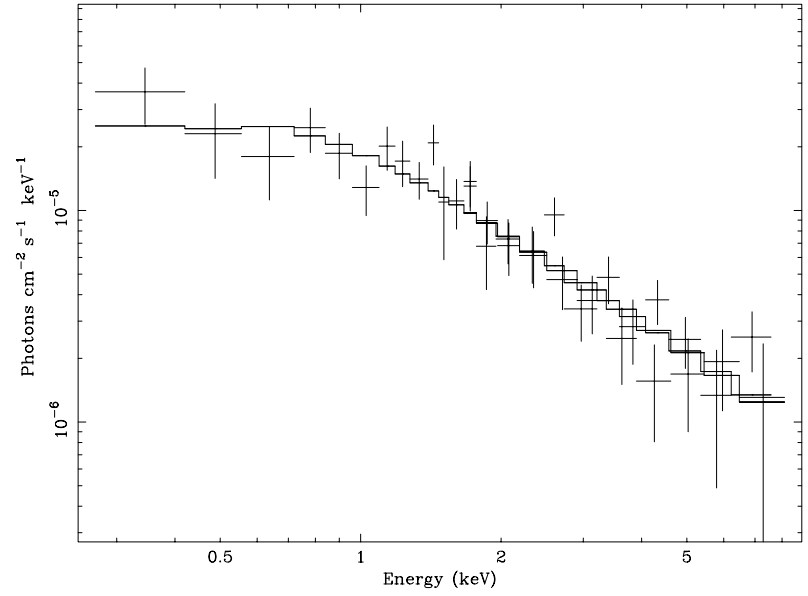

Fig. 6. The spectrum of the source 52, in the PSR J0751+1807 field of view, fitted with a power law model. The fit parameters can be found in Table 1.

spectrum, which cannot be fitted with a black body. We have also investigated temporal variation, binning the data into $1 \mathrm{ks}$ and $5 \mathrm{ks}$ bins. However, we find no significant variation during our observation. From the X-ray spectral fitting and short time scale temporal analysis, we cannot support nor disregard the galaxy hypothesis.

\section{PSR J1012+5307}

\subsection{Spectral analysis}

Due to the observation of PSR J1012+5307 being affected by a soft proton flare during the whole of the observation, we detect only one source in the field of view. This source is at the centre, with the same coordinates as those of PSR J1012+5307 and is therefore supposed to be the MSP PSR J1012+5307. We have not carried out any filtering for periods of higher background, as the whole observation would be included. Thus the signal to noise is poor for this observation.

We extracted the spectra in the same way as described in Sect. 3. We tried to fit simple models to the combined PN and MOS spectra. We find the model fits as given in Table 1 for the spectrum between $0.2-10.0 \mathrm{keV}$, when the $N_{\mathrm{H}}$ was frozen at $7 \times$ $10^{19} \mathrm{~cm}^{-2}$ (see Snowden et al. 1994). We cannot discriminate which of these fits is the best, however we present the spectrum of PSR J1012+5307 with a power law fit in Fig. 7. Allowing the $N_{\mathrm{H}}$ to vary gives values compatible with the above values. We determine an unabsorbed flux of $1.2 \times 10^{-13} \mathrm{ergs} \mathrm{cm}^{-2} \mathrm{~s}^{-1}$ $(0.2-10.0 \mathrm{keV})$.

\subsection{Timing analysis}

We corrected the timing data, as before, for the orbital movement of the pulsar and the data were folded on new radio ephemerides calculated to be correct for our observation (see Table 4), taking into account the time-delays due to the orbital motion. We used the data between $0.6-5.0 \mathrm{keV}$, as the signal-to-noise was best in this band. From the spectral fitting (see Sect. 4.1) we found that the majority of the 


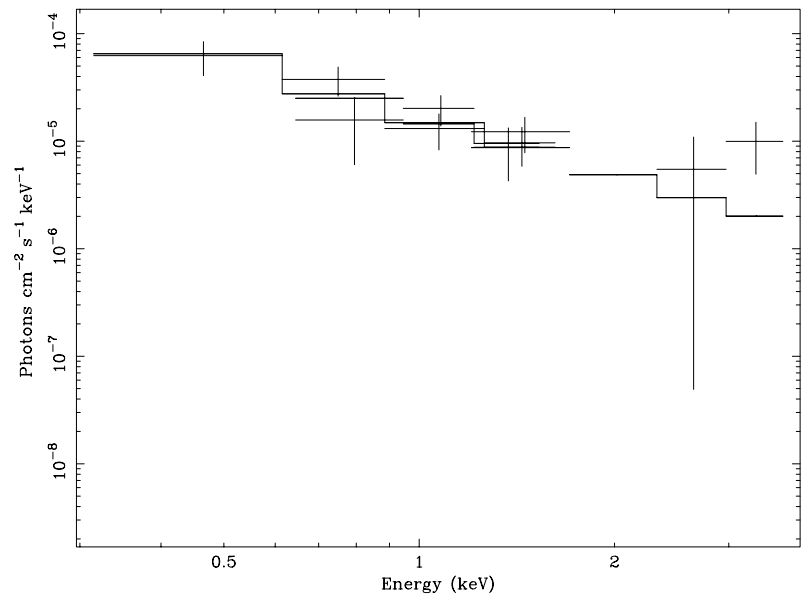

Fig. 7. The combined MOS and PN spectrum of PSR J1012+5307 fitted with a power law model. The fit parameters can be found in Table 1 .

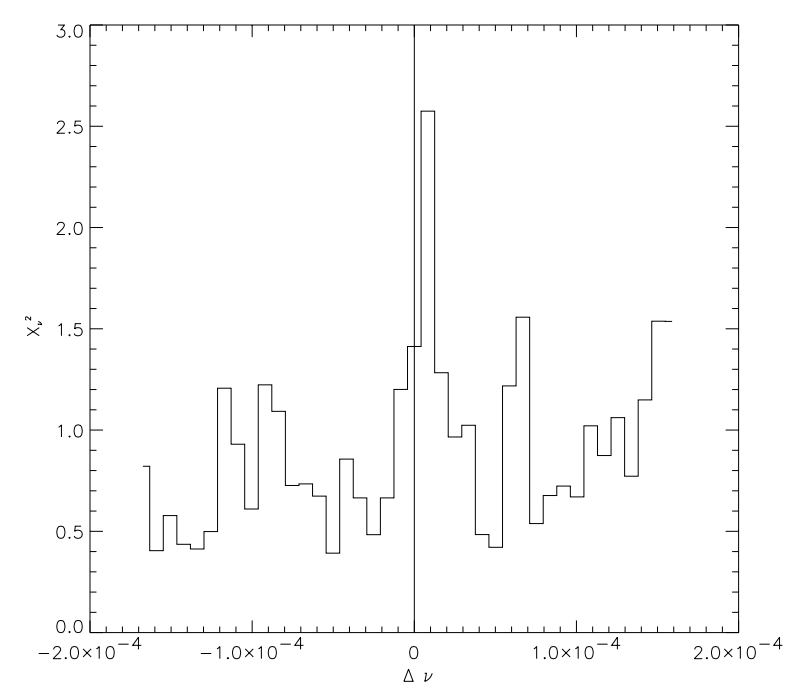

Fig. 8. $\chi_{v}^{2}$ versus change in frequency from the expected pulsation frequency (shown as the solid vertical line at $\Delta f=0.0$ ) for PSR J1012+5307.

emission from PSR J1012+5307 was in this energy band. We tested the hypothesis that there is no pulsation in the MSP PSR J1012+5307. The largest peak in the $\chi_{v}^{2}$ versus change in frequency from the expected frequency is at $\Delta v \simeq 8 \times$ $10^{-6} \mathrm{~s}^{-1}$, see Fig. 8. This implies a $\Delta f / f(=\Delta P / P)$ of $\sim 4 \times 10^{-8}$, similar to the value that we found for PSR J0218+4232 (Webb et al. 2004) $\left(\sim 1 \times 10^{-8}\right)$ and approaching the value of $<10^{-9}$ determined from the analysis of two revolutions of data of the MSP Crab (Kirsch et al. 2003; Kirsch 2003). It is also well inside the resolution of this dataset $\left(\sim 1 / T_{\text {obs }}\right)$, which is $7 \times 10^{-5} \mathrm{~s}^{-1}$, thus we can conclude that the data reduction and analysis made on the dataset are reliable. We find that the peak is significant at $3 \sigma$ (Buccheri et al. 1985). We folded the data on the radio period given in Table 4 . The folded lightcurve (0.6$5.0 \mathrm{keV}$ ), counts versus phase, is shown in the top panel of Fig. 9. We find some evidence for two pulses per period. Fitting the lightcurve with two Lorentzians (as Kuiper et al. 2002a) we find that the FWHM of the pulses is $\delta \phi_{1}=0.10 \pm 0.07$, centred at phase $\phi_{1}=0.36 \pm 0.04$ and $\delta \phi_{2}=0.09 \pm 0.03$, centred

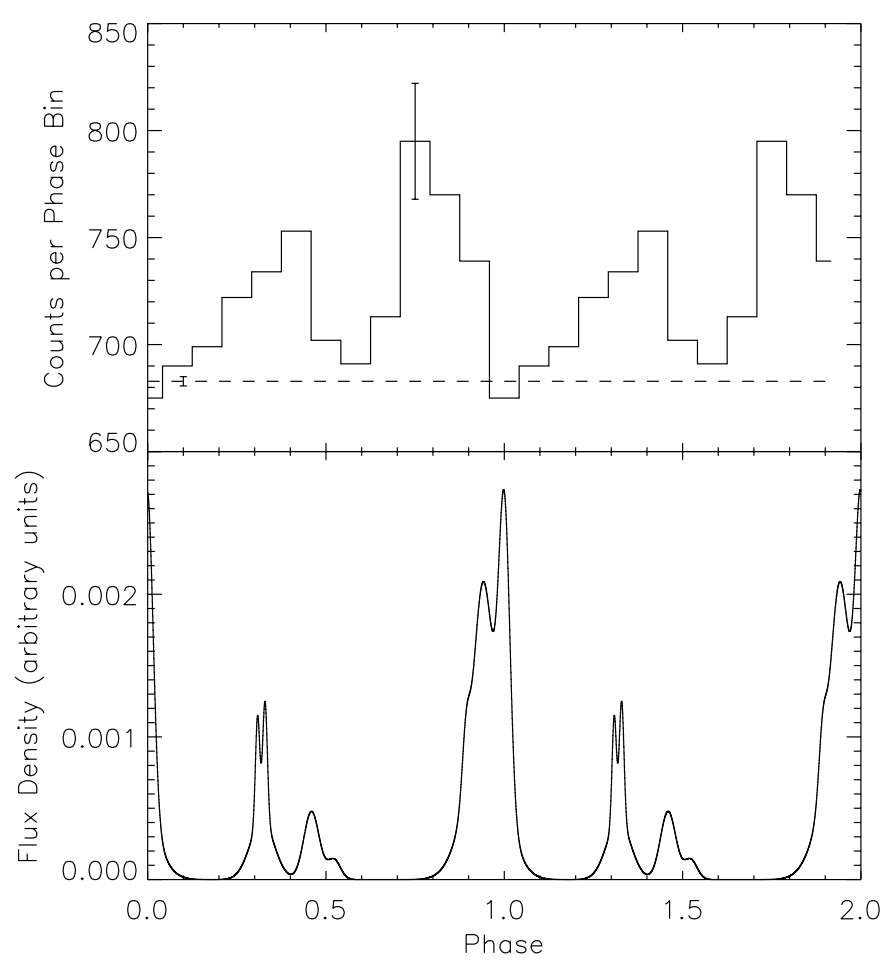

Fig. 9. Upper panel: lightcurve folded on the radio ephemeris and binned into 12 bins, each of $0.44 \mathrm{~ms}$. Two cycles are shown for clarity. A typical $\pm 1 \sigma$ error bar is shown. The dashed line shows the background level, where the error bar represents the $\pm 1 \sigma$ error. Lower panel: radio profile from the Effelsberg and the Lovell radio timing data. Again two cycles are shown for clarity.

Table 4. Ephemeris of PSR J1012+5307 from the Effelsberg and the Lovell radio timing data. Errors on the last digits are shown in parenthesis after the values.

\begin{tabular}{ll}
\hline \hline Parameter & Value \\
\hline Right ascension $(\mathrm{J} 2000)$ & $10^{\mathrm{h}} 12^{\mathrm{m}} 33^{\mathrm{s}} .43463677$ \\
Declination $(\mathrm{J} 2000)$ & $53^{\circ} 07^{\prime} 02^{\prime \prime} 4965199$ \\
Period $(P)$ & $0.00525605523 \mathrm{~s}$ \\
Period derivative $(\dot{P})$ & $0.726912 \times 10^{-20} \mathrm{~s} \mathrm{~s}^{-1}$ \\
Second period derivative $(\ddot{P})$ & $2.48928 \times 10^{-30} \mathrm{~s} \mathrm{~s}^{-2}$ \\
Frequency $(v)$ & $190.267837551251880(508) \mathrm{Hz}$ \\
Frequency derivative $(\dot{v})$ & $-6.200023(202) \times 10^{-16} \mathrm{~Hz} \mathrm{~s}^{-1}$ \\
Second frequency derivative $(\dot{v})$ & $1.871(294) \times 10^{-27} \mathrm{~Hz} \mathrm{~s}^{-2}$ \\
Epoch of the period (MJD) & 52018.324635450415 \\
Orbital period & $52243.7224464(17) \mathrm{s}$ \\
$a$. sin $i$ & $0.581817416(121)$ \\
Eccentricity & $<1.3 \times 10^{-6}$ \\
Time of ascending node $(\mathrm{MJD})$ & $52018.268144542(23)$ \\
\hline
\end{tabular}

at phase $\phi_{2}=0.80 \pm 0.03$. Fitting with two Gaussians gives similar results. Using a $Z_{4}^{2}$ test (Buccheri et al. 1983) on the $\mathrm{X}$-ray data, we determine a value of 13 , which corresponds to a probability of 0.99 that the pulse-phase distribution deviates from a statistically flat distribution. We find a pulsed fraction of $77 \pm 13 \%$.

In the lower panel in Fig. 9 we have plotted the radio lightcurve from the timing observations taken using the Effelsberg and Lovell telescopes. 


\section{Discussion}

We have investigated the two faint millisecond pulsars PSR J0751+1807 and PSR J1012+5307 in the X-ray band $0.2-10.0 \mathrm{keV}$, to ascertain the nature of the X-ray spectra. There are only a handful of MSPs that have been seen to pulse in both the radio and in X-rays to date (Becker \& Aschenbach 2002), thus we have also investigated whether these two MSPs show pulsations in X-rays.

Saito et al. (1997) showed that the MSP PSR B1821-24 has a similar magnetic field value, at the light cylinder radius $\left(B_{\mathrm{L}}\right)$, as the Crab pulsar. If this value can be taken to indicate the high-energy magnetospheric activity, they state that one can expect to see similar pulses from pulsars with a similar value of $B_{\mathrm{L}}$ and thus such pulsars are good candidates amongst the MSPs in which to look for magnetospheric X-ray pulsation in high energy bands. We have calculated the $B_{\mathrm{L}}$ for PSR J0751+1807 and PSR J1012+5307, using the values for the magnetic field strength from Zhang \& Harding (2000). We find $8.3 \times 10^{4}$ and $3.5 \times 10^{4} \mathrm{G}$ respectively. The value calculated for PSR J0751+1807 indicates that it is amongst the top 10 values for a millisecond pulsar. That of PSR J1012+5307 places it in the top half. Thus if a high $B_{\mathrm{L}}$ indicates high-energy magnetospheric activity this adds support to the detection of X-ray pulsations in our observation of PSR J0751+1807 and possibly to that of PSR J1012+5307.

We have also calculated the spin down energy $(\dot{E})$ and the luminosity in the $0.1-2.4 \mathrm{keV}$ band of these two pulsars, to compare the results with the correlation found between these two parameters by Becker \& Trümper (1997). Becker \& Trümper (1997) suggest that pulsars that obey this relationship emit X-rays produced by magnetospheric emission, originating from the co-rotating magnetosphere. For PSR J0751+1807 we find $\log (\dot{E})$ of 34.35 . To calculate the luminosity, we have used the distance calculated using the radio dispersion measure and the model of Taylor \& Cordes (1993). This gives a value of $\log \left(L_{\mathrm{x}}\right)$ of $31.0 \pm 0.2$, which places the point close to the expected value of 31.2 using the correlation proposed by Becker \& Trümper (1997). However, using the more recent model of Cordes et al. (2003a,b) diminishes the distance by almost a factor of 2 , to $1.1 \mathrm{kpc}$. This gives a $\log \left(L_{\mathrm{x}}\right)$ of $30.5 \pm 0.2$ and thus displaces the point further from the expected value. For the MSP PSR J1012+5307 we find a $\log (\dot{E})$ of 34.2 and a $\log \left(L_{\mathrm{x}}\right)$ of $30.4 \pm 0.3$, where the expected $\log \left(L_{\mathrm{X}}\right)$ is approximately 31.1. This places the point some distance from the expected value, which may indicate that the relationship is not a hard and fast rule. Indeed Pavlov et al. (2002) found recently that when analysing pulsars in the $2.0-10.0 \mathrm{keV}$ band, this relationship does not hold true.

\subsection{PSR J0751+1807}

We find that the best fitting model to the X-ray spectrum of PSR J0751+1807 is a power law. This is indicative of a magnetospheric origin of the X-ray emission. We have shown evidence for a single broad pulse emitted from this MSP, where the pulsation appears to change only slightly with increasing energy (becomes slightly broader). The observations also indicate that the pulsed fraction possibly increases at higher energies. A broad pulsation can be observed from pulsars showing hard magnetospheric emission or soft thermal emission and thus we cannot yet discern the origin of the X-ray emission from PSR J0751+1807 with this data set. Indeed this pulsar may be best fitted by a multi-component model, as is the case with several other brighter MSPs e.g. PSR J0218+4232, (Webb et al. 2004) or PSR J0437-4715, (Zavlin \& Pavlov 1998; Zavlin et al. 2002). Longer observations of PSR J0751+1807 will help to distinguish the true nature of the spectrum.

\subsection{PSR J1012+5307}

We cannot discriminate which of the model fits to the MSP PSR J1012+5307 is the best, however we find that the single power law has a similar photon index to the X-ray spectrum of PSR J0751+1807 (see Sect. 3.1), which could indicate a magnetospheric origin of the X-ray emission. However, the temperature of the blackbody $\left(1.9 \pm 0.5 \times 10^{6} \mathrm{~K}\right)$ is consistent with that emitted from the heated polar caps of a millisecond pulsar $\left(10^{6}-10^{7} \mathrm{~K}\right.$ e.g. Zhang \& Cheng 2003; Zavlin \& Pavlov 1998, and references therein). Calculating the radius of the emission area from the blackbody model fit, we find a radius of $0.05 \pm_{0.02}^{0.01} \mathrm{~km}$, which is smaller than the expected radius of emission from polar caps $(\sim 1 \mathrm{~km}$ e.g. Zhang \& Cheng 2003; Zavlin \& Pavlov 1998, and references therein). Zavlin et al. (1996) state however, that spectral fits with simplified blackbody models can produce higher temperatures and smaller sizes due to the fact that the X-ray spectra emerging from light-element atmospheres are harder than blackbody spectra. Alternatively, Zavlin \& Pavlov (1998) and Zavlin et al. (2002) suggest that the thermal emission can be from non uniform polar caps and we may therefore be seeing the emission from the hotter central region of the caps.

Folding the timing data on the radio frequency, we find evidence for two pulses emitted from this MSP, separated by approximately 0.4 in phase. This is similar to other millisecond pulsars, e.g. PSR J0218+4232 (Webb et al. 2004) and PSR B1821-24 (Saito et al. 1997).

\section{Conclusions}

We have presented XMM-Newton data of the faint millisecond pulsars PSR J0751+1807 and PSR J1012+5307. Both of these pulsars have a reasonably large magnetic field at the light cylinder radius, which could indicate that both of these MSPs should show pulsations in X-rays. We present for the first time the X-ray spectra of these two faint millisecond pulsars. We find that a power law model best fits the spectrum of PSR J0751+1807, $\Gamma=1.59 \pm 0.20$, with an unabsorbed flux of $4.4 \times 10^{-14}$ ergs cm $\mathrm{cm}^{-2} \mathrm{~s}^{-1}(0.2-10.0 \mathrm{keV})$. A power law is also a good description of the spectrum of PSR J1012+5307, $\Gamma=1.78 \pm 0.36$, with an unabsorbed flux of $1.2 \times 10^{-13} \mathrm{ergs} \mathrm{cm}^{-2} \mathrm{~s}^{-1}(0.2-10.0 \mathrm{keV})$. However, a blackbody model cannot be excluded as the best fit to the data. We have also shown evidence to suggest that both of these MSPs show X-ray pulsations. PSR J0751+1807 appears to show a 
single pulse, whereas PSR J1012+5307 may show some evidence of two pulses per pulse period.

Acknowledgements. We wish to thank A. Marcowith for his advice pertaining to the work in this manuscript. This article was based on observations obtained with XMM-Newton, an ESA science mission with instruments and contributions directly funded by ESA Member States and NASA. The authors also acknowledge the CNES for its support in this research.

\section{References}

Barcons, X., Carrera, F. J., Watson, M. G., et al. 2002, A\&A, 382, 522 Becker, W., \& Trümper, J. 1993, Nature, 365, 528

Becker, W., Trümper, J., \& Lundgren, S. C. 1996, MNRAS, 282, L33

Becker, W., \& Trümper, J. 1997, A\&A, 326, 682

Becker, W., \& Aschenbach, B. 2002, Proc. of the 270. WE-Heraeus Seminar on Neutron Stars, Pulsars, and Supernova Remnants, ed. W. Becker, H. Lesch, \& J. Trümper, MPE Report, 278

Buccheri, R., Bennett, K., Bignami, G., et al. 1983, A\&A, 128, 245

Buccheri, R., Sacco, B., Damico, N., \& Hermsen, W. 1985, Nature, 316, 131

Callanan, P. J., Garnavich, P. M., \& Koester, D. 1998, MNRAS, 298, 207

Cordes, J. M., Joseph, T., \& Lazio, W. 2003a, ApJ, submitted

Cordes, J. M., Joseph, T., \& Lazio, W. 2003b, ApJ, submitted

Halpern, J. P. 1996, ApJ, 459, L9

Halpern, J. P., \& Wang, F. Y.-H. 1997, BAAS, 29, 1391

Hasinger, G., Altieri, B., Arnaud, M., et al. 2001, A\&A, 365, L45

Jansen, F., Lumb, D., Altieri, B., et al. 2001, A\&A, 365, L1-6

Kirsch, M. and the EPIC Consortium, 2002, XMM-SOC-CAL-TN0018
Kirsch, M. G. F., Becker, W., Benlloch-Garcia, S., et al. 2003, Proc. SPIE, 5165

Kirsch, M. G. F. 2003, Ph.D. Thesis, Univ. of Tübingen, ISBN3-89959-070-8

Kuiper, L., Hermsen, W., Verbunt, F., et al. 2000, A\&A, 359, 615

Kuiper, L., Hermsen, W., Verbunt, F., et al. 2002a, ApJ, 577, 917

Kuiper, L., \& Hermsen, W. 2002b, The Gamma-Ray Universe, ed. A. Goldwurm, D. Neumann, J. Tran, \& V. Thanh (Vietnam: The Gioi Publishers)

Kuster, M., Kendziorra, M., Benlloch, S., et al. 2002, New Visions of the Universe in the XMM-Newton and Chandra Era, ed. F. Jansen, ESA-SP 488

Lange, Ch., Camilo, F., Wex, N., et al. 2001, MNRAS, 326, 274

Lundgren, S. C., Zepka, A. F., Cordes, J. M., et al. 1993, AA\&S, 183, 3703

Lundgren, S. C., Zepka, A. F., \& Cordes, J. M. 1995, ApJ, 453, 419

Nicastro, L., Lyne, A. G., Lorimer, D. R., et al. 1995, MNRAS, 273, L68

Possenti, A., Cerutti, R., Colpi, M., \& Mereghetti, S. 2002, A\&A, 387, 993

Saito, Y., Kawai, N., Kamae, T., et al. 1997, ApJ, 477, 37

Stark, A. A., Gammie, C. F., Wilson, R. W., et al. 1992, ApJS, 79, 77

Snowden, S. L., Hasinger, G., Jahoda, K., et al. 1994, ApJ, 430, 601

Strüder, L., Briel, U., Dennerl, K., et al. 2001, A\&A, 365, L18

Taylor, J. H., \& Cordes, J. M. 1993, ApJ, 411, 674

Turner, M. J. L., Abbey, A., Arnaud, M., et al. 2001, A\&A, 365, L27

Webb, N. A., Olive, J.-F., \& Barret, D. 2004, A\&A, 417, 181

Zavlin, V. E., Pavlov, G. G., \& Shibanov, Y. A. 1996, A\&A, 315, 141

Zavlin, V. E., \& Pavlov, G. G. 1998, A\&A, 329, 583

Zavlin, V. E., Pavlov, G. G., Sanwal, D., et al. 2002, ApJ, 569, 894

Zhang, B., \& Harding, A. 2000, ApJ, 532, 1150

Zhang, L., \& Cheng, K. S. 2003, A\&A, 398, 639 